\title{
WATER LEVEL FLUCTUATION USING SURVEILLANCE CAMERA
}

\author{
Nur Atirah Muhadi ${ }^{1, *}$, Ahmad Fikri Abdullah ${ }^{1,2}$, Siti Khairunniza Bejo ${ }^{1}$, Muhammad Razif Mahadi ${ }^{1}$, Ana Mijic ${ }^{3}$ \\ ${ }^{1}$ Department of Biological and Agricultural Engineering, Faculty of Engineering, Universiti Putra Malaysia, 43400 Serdang, \\ Selangor, Malaysia \\ ${ }^{2}$ Institute of Aquaculture and Aquatic Sciences, Batu 7, Jalan Kemang 6, Teluk Kemang, 71050 Si Rusa, Port Dickson, Negeri \\ Sembilan., Malaysia \\ ${ }^{3}$ Department of Civil and Environmental Engineering, Imperial College London, South Kensington Campus, London SW7 2AZ, UK
}

Commission 4, WG 7

KEY WORDS: Hybrid technique, Image segmentation, Water level fluctuation, Water segmentation, Surveillance camera

\begin{abstract}
Floods are the most frequent type of natural disaster that cause loss of life and damages to personal property and eventually affect the economic state of the country. Researchers around the world have been made significant efforts in dealing with the flood issue. Computer vision is one of the common approaches being employed which include the use of image segmentation techniques for image understanding and image analysis. The technique has been used in various fields including in flood disaster applications. This paper explores the use of a hybrid segmentation technique in detecting water regions from surveillance images and introduces a flood index calculation to study water level fluctuations. The flood index was evaluated by comparing the result with water level measured by sensor on-site. The experimental results demonstrated that the flood index reflects the trend of water levels of the river. Thus, the proposed technique can be used in detecting water regions and monitoring the water level fluctuation of the river.
\end{abstract}

\section{INTRODUCTION}

Flood frequency and flood impacts have worsened with the extreme changes in climate events, affecting more than 1.5 billion people worldwide from 2000 to 2019 (UNDRR, 2020). Therefore, it is important to monitor and detect the occurrence of flood events by improving the flood detection and monitoring systems so that the severity of flood impact can be reduced. A common approach is by using computer vision, where the images are captured and processed using image processing techniques.

Image segmentation plays an important role in image processing and computer vision applications. Image segmentation is the process of partitioning an image into multiple regions, often based on similar characteristics of the pixels in the image to obtain useful information from surveillance images. It has been applied in the fields of medical imaging, automated driving as well as in water management practices such as in flood monitoring applications (Feng et al., 2019; Jena et al., 2018; Kim \& Kim, 2003; Ko \& Kwak, 2012; Popescu et al., 2015; Yuan et al., 2017). Previous researchers have developed various image segmentation techniques including thresholding, boundary-based, region-based, hybrid technique, and many more.

There have been several papers discussing image segmentation methods that are specifically applied to flood disasters. Lai et al. (2007) proposed to use a thresholding method to identify and detect flood disaster events by analyzing the color information and texture. Other researchers that used these same characteristics to identify flood events are Borges, et al, (2008), San Miguel \& Ruiz Jr., (2013), and Filonenko et al., (2015). Besides, Lo et al. (2015) practiced the region growing method for flood region detection because region growing is more suitable for a dynamic background.
Besides, Jyh-Horng et al. (2015) suggested using mean-shift and region growing methods for automated identification methods for flood monitoring. On the other hand, Geetha et al. (2018) utilized crowdsourced images to detect the extent of flooding area using a color-based threshold to segment the floodwater, which was based on the brown color intensity. Next, Langhammer and Vacková (2018) exploited the seed region growing algorithm to perform object-based image segmentation to classify the image into several categories.

To date, no general solution has been found to solve the image segmentation problems in order to achieve reliable accuracy for any applications including flood disaster applications. However, previous studies reported that the hybrid technique, a combination of two or more segmentation methods, has more advantages than using an individual segmentation method (Khan, 2014; Lankton et al., 2007; Muhadi et al., 2020; Singh \& Misra, 2017).

Therefore, this paper demonstrates the use of the hybrid technique for water segmentation tasks. For water level fluctuation, a flood index was introduced with the same concept as the one proposed by Moy de Vitry et al. (2019). To evaluate the performance of the flood index, the results were compared with water level measured by sensor on-site.

\section{METHODOLOGY}

This study consists of two processes, the first was the segmentation process and the second process was the flood index computation process. Both processing steps were performed using MATLAB 9.10 (R2021a) on a notebook equipped with a $2.60 \mathrm{GHz}$ Intel ${ }^{\circledR}$ Core $^{\mathrm{TM}}$ i7 $\mathrm{CPU}$ and $16 \mathrm{~GB}$ RAM. 


\subsection{Hybrid Segmentation Method}

A single method of image segmentation could not be used for all types of images and all image segmentation methods could not perform well for one particular image (Muhadi et al., 2020). Therefore, a hybrid technique, which consists of multiple methods of image segmentation could improve the segmentation results (Khan, 2014). This paper adopted the hybrid technique proposed by Lankton et al. (2007) to detect the flooding area and more detailed information can be found in the original work. Basically, the authors presented an idea of combining geodesic active contours and region-based active contours. The segmentation task began from the initial curve (Figure 1) and proceeded to each point on the curve. The image is segmented by modeling the nearby points inside and outside of the object using mean intensities of the local regions at each point on the true edge of the object. The suggested method was used to segment image that has a weak homogeneous intensity profile and poor edge definition. The original image was resized to a smaller size from a resolution of $720 \times 960$ to $144 \times 256$ for fast computation results.

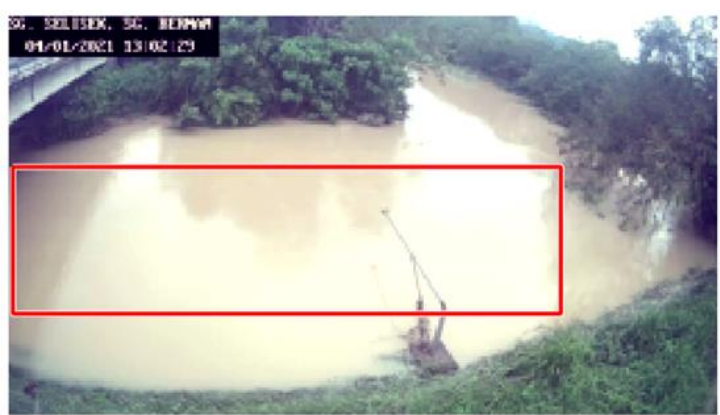

Figure 1. The curve initialization for the segmentation process.

\subsection{Flood Index Computation}

The image that has been segmented was then converted into a binary image for water level analysis. Water level fluctuation can be portrayed by a flood index, a ratio of the visible flooded area over a total area. In this study, a region of interest (ROI) was defined prior to when the river was in normal condition and the same ROI was used for every image used in this experiment. The ROI was located outside the riverbank so that water overflow can be detected by identifying pixels that were previously classified as background that has changed into water class when the water level rose. The ROI was selected in the area that was away from any objects that might cause reflection problems and lead to segmentation errors. For this particular location, the rectangular ROI was located at an image coordinate of $[40,116]$ whose length and width are $58 \times 28$ as shown in Figure 2.

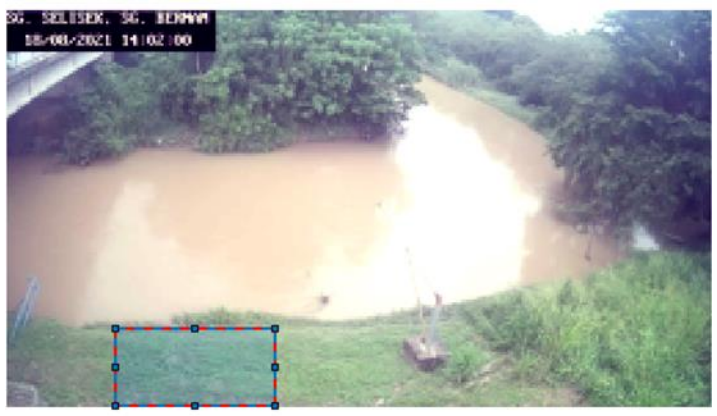

(a)

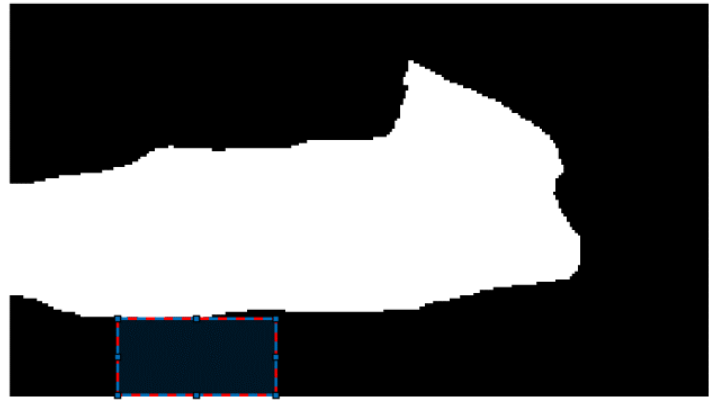

(b)

Figure 2. The ROI of the study area. (a) ROI overlaid with the original image. (b) ROI overlaid with the binary image. When in normal condition, the whole ROI is filled with black pixels

(no water).

The flood index ranges from $0 \%$ to $100 \%$, where $0 \%$ means no flooding and $100 \%$ indicates that the area is completely flooded. The flood index of the defined ROI during normal water level was 0 . As the water level rises, the flood index is also increased and when the water level drops to the normal level, the flood index scores 0 . The water level fluctuation was described by the following equation:

$$
\text { Flood index }=\frac{\text { Water pixels }}{\text { ToI }} \times 100,
$$

\section{RESULTS AND DISCUSSION}

In general, this study used images from a surveillance camera that was installed nearby the river. The image dataset was acquired in December 2020 and the early year of 2021.

\subsection{Hybrid segmentation results}

Before the segmentation process took place, the original images were pre-processed by adjusting the color intensity of the image to enhance the color contrast for better segmentation results. Figure 3 shows one of the outputs from the segmentation task. The image shows that some parts of the water region were failed to be classified correctly. However, because the ROI had been defined at a definite area with specific criteria, the segmentation error would not affect the flood index results.

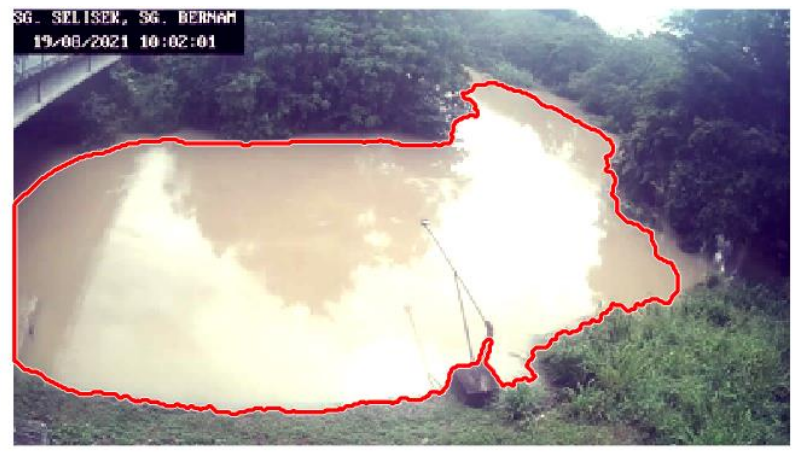

Figure 3. The segmentation result that extracts water from the background.

\subsection{Water level fluctuations}

Results from the segmentation process were converted into binary images. The flood index was computed by calculating 
the flooded pixels (white pixels) within the ROI as shown in Figure 4.

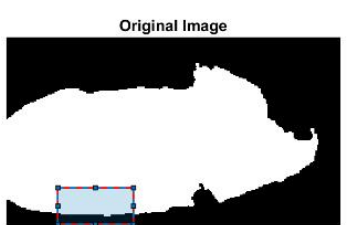

(a)

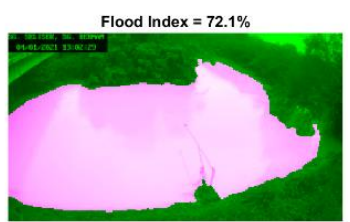

(b)
Figure 4. The flood index for the image of 4th of August 2021.

(a) The ROI contains more white pixels than black. (b) The flood index computed was $72.1 \%$.

The segmentation results were then used to calculate the flood index. Each image in the image dataset was segmented and the flood index for each image was computed. Figure 5 displays the flood index for all image datasets. For this particular area, the normal water level was between 23 to 26 meters, where the flood index of these ranges should be 0 . The flood index values vary depending on the water level during the particular time.
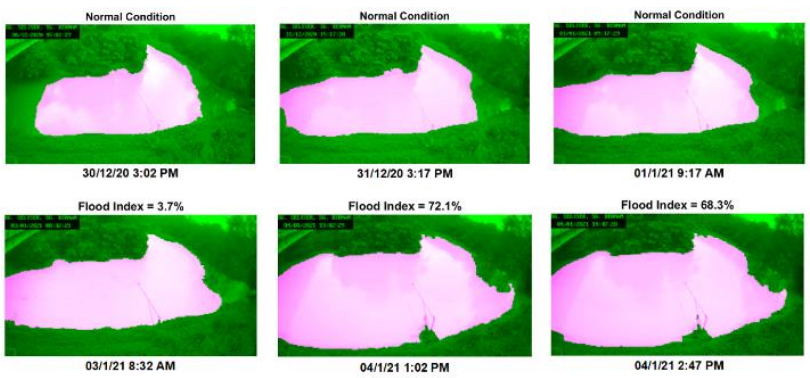

Figure 5. Flood index for all images used in this experiment.

To determine the reliability of the flood index, the results were compared with the water level measured by the sensor in the station as shown in Figure. The graph illustrates the there is a strong correlation between flood index and water level in a nonlinear form. This may be due to the uneven topography of the flooded area. Besides, it can be seen from the graph that the flood index follows the trend of water level, hence it reflects the water level fluctuation of the area.

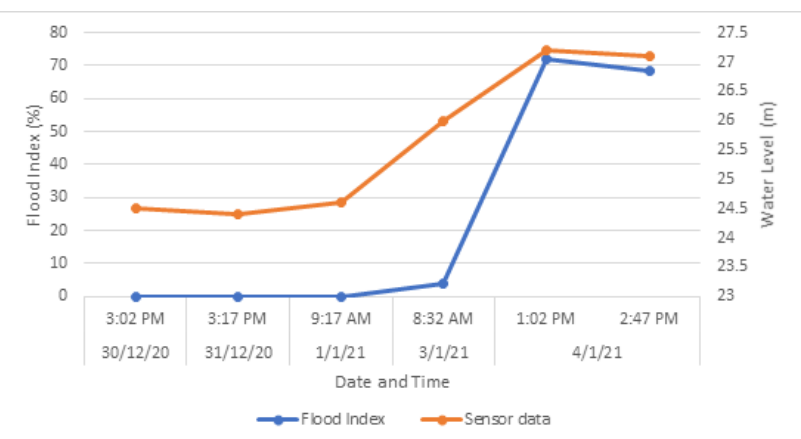

Figure 6. Flood index (blue line) and sensor data (orange) were plotted against the local time of the images used.

In particular, the axis on the left-hand side displays the flood index value for the blue line, while the axis on the right-hand side represents the values of the water level measured by the sensor (orange line). The graph shows that the value of the flood index for 30th and 31st December 2020, and 1st January 2021 were 0 . The water levels during these times were less than 25 meters, which is within the normal level range. Hence, the ROI was completely dry and not flooded by water. However, on the 3rd January, the flood index rose to $3.7 \%$, which means that there were some changes of class pixels in the defined ROI. It is found that during this time, the water level was 26 meters, which almost exceed the normal level threshold and the water level was slightly increased in terms of flood extent. On the 4th of January, the water levels were significantly increased to more than 27 meters, which caused the increments in the flood index. The flood index values for both images during that day were more than $50 \%$ which indicates that more than half of the ROI was submerged.

\section{CONCLUSION}

Information on floodwater during flood events could help the authorities to monitor and assist in decision-making during flood events. In this paper, a hybrid segmentation technique was used to extract the water information from the still images. A flood index calculation was then applied to study the water level fluctuation from the segmentation results. The findings demonstrated that the flood index reflects the trend of water levels of the river. In summary, the implementation of the proposed technique will provide invaluable information, especially in water region detection and monitoring the fluctuation of the river, which can be useful in decision making or coordinating flood relief operations. A similar study to identify water regions from surveillance images has been performed using deep learning semantic segmentation (Muhadi et al., 2021). Future work can be done by adopting different neural network architectures to find out which architecture performs well for water-related problems.

\section{ACKNOWLEDGEMENTS}

This study was funded by Universiti Putra Malaysia under the Putra Grant, GPB (Grant number: 9678700). The authors appreciate the support for this study from the Universiti Putra Malaysia and the Institute of Aquaculture and Aquatic Sciences. The authors would like to thank the Department of Irrigation and Drainage for sharing the surveillance images and water level data publicly.

\section{REFERENCES}

Borges, P. V. K., Mayer, J., \& Izquierdo, E. (2008). A probabilistic model for flood detection in video sequences. 2008 15th IEEE International Conference on Image Processing, 1316. https://doi.org/10.1109/ICIP.2008.4711679

Feng, D., Haase-Schuetz, C., Rosenbaum, L., Hertlein, H., Glaeser, C., Timm, F., Wiesbeck, W., \& Dietmayer, K. (2019). Deep Multi-modal Object Detection and Semantic Segmentation for Autonomous Driving: Datasets, Methods, and Challenges. http://arxiv.org/abs/1902.07830

Filonenko, A., Wahyono, Hernandez, D. C. C., Seo, D., \& Jo, K. H. (2015). Real-time flood detection for video surveillance. IECON 2015 - 41st Annual Conference of the IEEE Industrial Electronics Society, 4082-4085.

Geetha, M., Manoj, M., Sarika, A. S., Mohan, M., \& Rao, S. N. (2017). Detection and estimation of the extent of flood from crowd sourced images. 2017 International Conference on Communication and Signal Processing (ICCSP), 603-608. https://doi.org/10.1109/ICCSP.2017.8286429 
Jena, M., Prava Mishra, S., \& Mishra, D. (2018). A survey on applications of machine learning techniques for medical image segmentation. International Journal of Engineering and Technology, 7(4), 4489-4495. https://doi.org/10.14419/ijet.v7i4.19005

Jyh-Horng, W., Chien-Hao, T., Lun-Chi, C., Shi-Wei, L., \& Fang-Pang, L. (2015). Automated Image Identification Method for Flood Disaster Monitoring In Riverine Environments: a Case Study in Taiwan. AASRI International Conference on Industrial Electronics and Applications (IEA 2015), June 2016, 268-271. https://doi.org/10.2991/iea-15.2015.65

Khan, M. W. (2014). A Survey: Image Segmentation Techniques. International Journal of Future Computer and Communication, 3(2), 89-93. https://doi.org/10.1007/978-98113-0761-4_105

Kim, J. B., \& Kim, H. J. (2003). Multiresolution-based watersheds for efficient image segmentation. Pattern Recognition Letters, 24(1-3), 473-488. https://doi.org/10.1016/S0167-8655(02)00270-2

Ko, B., \& Kwak, S. (2012). Survey of computer vision-based natural disaster warning systems. Optical Engineering, 51(7), 070901. https://doi.org/10.1117/1.oe.51.7.070901

Lai, C. L., Yang, J. C., \& Chen, Y. H. (2007). A real time video processing based surveillance system for early fire and flood detection. 2007 IEEE Instrumentation \& Measurement Technology Conference IMTC 2007, 1-6. https://doi.org/10.1109/imtc.2007.379190

Langhammer, J., \& Vacková, T. (2018). Detection and Mapping of the Geomorphic Effects of Flooding Using UAV Photogrammetry. Pure and Applied Geophysics, 175(9), 32233245. https://doi.org/10.1007/s00024-018-1874-1

Lankton, S., Nain, D., Yezzi, A., \& Tannenbaum, A. (2007). Hybrid geodesic region-based curve evolutions for image segmentation. Medical Imaging 2007: Physics of Medical Imaging, 6510(d), 65104U. https://doi.org/10.1117/12.709700

Lo, S.-W. W., Wu, J.-H. H., Lin, F.-P. P., \& Hsu, C.-H. H. (2015). Cyber surveillance for flood disasters. Sensors (Switzerland), 15(2), 2369-2387. https://doi.org/10.3390/s150202369

Moy de Vitry, M., Kramer, S., Wegner, J. D., \& Leitão, J. P. (2019). Scalable Flood Level Trend Monitoring with Surveillance Cameras using a Deep Convolutional Neural Network. Hydrology and Earth System Sciences Discussions, February, 1-21. https://doi.org/10.5194/hess-2018-570

Muhadi NA, Abdullah AF, Bejo SK, Mahadi MR, Mijic A. (2021). Deep Learning Semantic Segmentation for Water Level Estimation Using Surveillance Camera. Applied Sciences, 11(20), 9691. https://doi.org/10.3390/app11209691

Muhadi, N. A., Abdullah, A. F., Bejo, S. K., Mahadi, M. R., \& Mijic, A. (2020). Image Segmentation Methods for Flood $\begin{array}{llll}\text { Monitoring System. Water, 12(6), } 1825 . & \end{array}$ https://doi.org/10.3390/w12061825

Popescu, D., Ichim, L., \& Caramihale, T. (2015). Flood areas detection based on UAV surveillance system. 2015 19th International Conference on System Theory, Control and
Computing, ICSTCC 2015 - Joint Conference SINTES 19, $\begin{array}{lllll}\text { SACCS } & 15, & \text { SIMSIS } & 19, & 753-758 .\end{array}$ https://doi.org/10.1109/ICSTCC.2015.7321384

San Miguel, M. J. P., \& Ruiz, C. R. (2016). A flood detection and warning system based on video content analysis. International Symposium on Visual Computing, 8034, 65-74. https://doi.org/10.1007/978-3-642-41939-3

Singh, V., \& Misra, A. K. (2017). Detection of plant leaf diseases using image segmentation and soft computing techniques. Information Processing in Agriculture, 4(1), 41-49. https://doi.org/10.1016/j.inpa.2016.10.005

UNDRR. (2020). The Human Cost of Disasters - An overview of the last 20 years 2000-2019. In Human Cost of Disasters. https://doi.org/10.18356/79b92774-en

Yuan, Y., Chao, M., \& Lo, Y. C. (2017). Automatic Skin Lesion Segmentation Using Deep Fully Convolutional Networks with Jaccard Distance. IEEE Transactions on Medical Imaging, $36(9)$, $1876-1886$

https://doi.org/10.1109/TMI.2017.2695227 